\title{
Smart System for Elderly Wandering Person
}

\author{
Vidya J, Vineeth R, P Sri Harsha, J Venkata Sai, Noor Mohammed
}

\begin{abstract}
Nowadays wandering became one of the frequent, ticklish and deleterious behaviors of elders suffering from dementia. The same may lead to consequences like getting lost or falling down. The proposed system concentrates on problems related to wandering. In this system, we are planning a security support system focused on android for identifying the location of a wandering elder person. This system is employed with the GPS that can detect the position of the individual within and around buildings, and transmit the location to the server device. It can also detect the sound of the drifting elder person and identify the accurate position. There will be an accelerometer which detects when a person falls. This model informs the caregiver by sending the notification.
\end{abstract}

Keywords : GPS, Accelerometer, Node MCU, Sound Sensor

\section{INTRODUCTION}

According to an estimate by the largest health organization, the 2019 population of elderly people over the age of 75 was nearly seven-four million around the world. Three million elderly people with Alzheimer's number and are expected to reach 4.5 million by 2030[1]. Alzheimer's is a chronic illness and it's very difficult to lead a normal lifestyle. We have many tic disorders such as wandering, inadequate verbal communication, being uncooperative and this can lead to a major cause of death, and caregivers have a particularly serious problem. The location and condition of the person drawing up must be tracked.

One of the main areas of focus is the health of the elderly, particularly those suffering from Alzheimer's [2]. Such people tend to cope with their habits but often they may suffer from material disorientation situations and even get lost in some cases. Tracking and monitoring tools have therefore emerged as a critical solution for supporting elderly people navigate their mobility problems.

Revised Manuscript Received on April 02, 2020.

* Correspondence Author

Vidya J*, Dept. of CSE, GITAM University, Bengaluru, India. Email: vjyothip@gitam.edu

Vineeth R, Dept. of CSE, GITAM University, Bengaluru, India. Email: rajavineeth25041999@gmail.com

P Sri Harsha, Dept. of CSE, GITAM University, Bengaluru, India. Email: sriharsha8296@gmail.com

J Venkata Sai, Dept. of CSE, GITAM University, Bengaluru, India. Email: venkatasai0078@gmail.com

Noor Mohammed, Dept. of CSE, GITAM University, Bengaluru, India. Email: shaiknoormohammed111@gmail.com

(C) The Authors. Published by Blue Eyes Intelligence Engineering and Sciences Publication (BEIESP). This is an open access article under the CC BY-NC-ND license (http://creativecommons.org/licenses/by-nc-nd/4.0/)

\section{RELATED WORK}

In recent years, Alzheimer's disease is considered a grave threat to most elderly adults. Our methodology proposes how we track and resolve the problems that were being faced by previous prototypes. Some of them being explained here. Researchers note that wandering happens among the majority of patients facing Alzheimer's because they have a convincing desire to walk and after a while they find it hard to go back as they suffer from memory loss [4]. We may assume that GPS is an established localization program but we need some other methods for any medical emergencies. One of Spadaro, Danielson, and Tyson's experiments used an Android framework that allows for a tracking system named I Wander. The unit was analyzed, along with GPS, with Bayesian theory to measure the patient's wandering probability [3]. Based on measured results, the I Wander could automatically alert the Alzheimer's patient and guide it to a safe place. It can be provided when any medical assistance is needed. But that version was limited to safer places for navigation only.

Abbate et al. created a wandering recognition device based on android phones, which provides a support service to help the elderly. It has been pointed out that the elderly can experience many fall-like movements, such as sitting on a sofa, lying on a bed, walking or running, and involuntarily hitting the monitoring system sensor[5]. Such fall-like behaviors can produce an impact in the magnitude of acceleration that is relatively similar to a true fall. To effectively distinguish fall-like events from real-fall accidents, Abbate et al. engaged a "machine learning theory" to a classification engine to enhance the results of fall detection[7]. The data suggest the unit demonstrates improved recognition capability in the identification of real-fall accidents through the training process.

\section{PROPOSED SYSTEM}

Using a lifesaving location app is one way to monitor seniors roaming around. This app allows seniors to be identified quickly using GPS tracking. The echo of the wandering elderly person can be heard. The sound is used to identify the person's location. There will be an accelerometer that senses when a person falls and sends alerts to the caregiver.

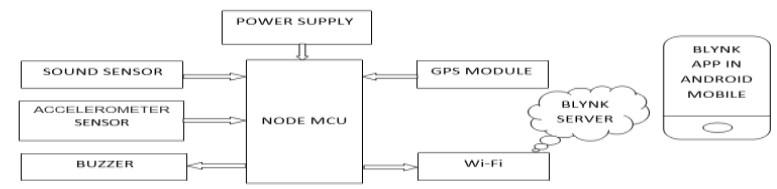

Fig 1: Proposed Device Architecture

Published By:

Blue Eyes Intelligence Engineering \& Sciences Publication

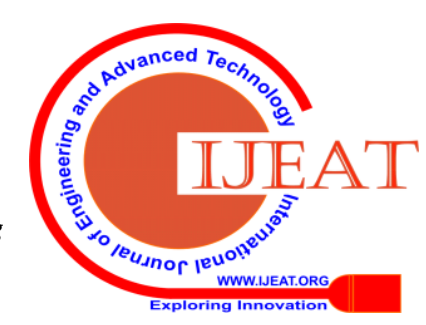




\section{Smart System for Elderly Wandering Person}

In the above Fig1 the components are explained below

\section{A.Node MCU}

In this framework, we are using a Node MCU with a microcontroller.

NodeMCU is a free firmware which offers open source prototyping board designs. "NodeMCU" name is a combination of "node" and "MCU" (micro controller unit). The term "NodeMCU" refers to firmware but not related buildout kits. It has a Wi-Fi module[6 ].

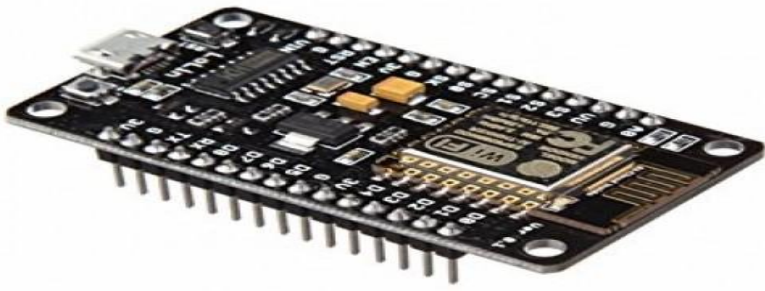

Fig 2: Node MCU

\section{B. Accelerometer}

The Accelerometer is an electronic-mechanical device used to evaluate the forces of acceleration. Such forces may sometimes be static, similar to the gravity constant force. In case of a large number of mobile devices, the movement and movements are active to hear. Acceleration is defined as determination of the transition occurring in velocity, or in case of time-divided velocity.

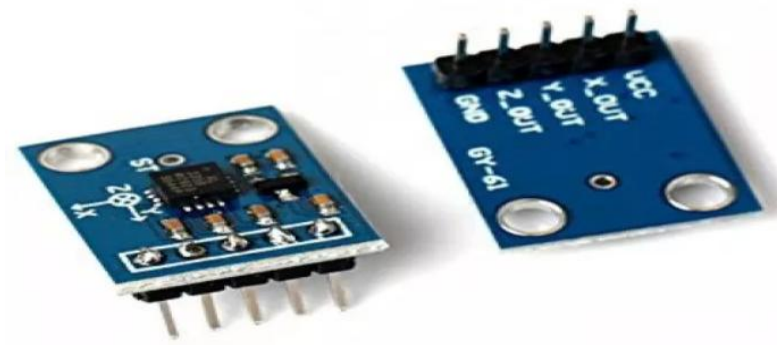

Fig 3: Accelerometer

\section{C.Sound Sensor}

The sound sensor is a kind of module used to detect the sound. Commonly, this device is used to detect the level of sound. This sensor consists of a microphone to provide the noise to buffer and further components [8]. This sensor detects the sound, and processes an output voltage signal to NODE MCU.

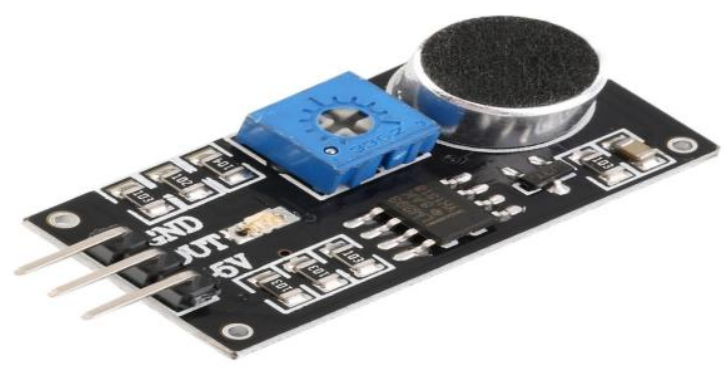

Fig 4: Sound Sensor

\section{D.GPS module}

Global positioning satellite is commonly used for location detection of the person carrying the prototype.

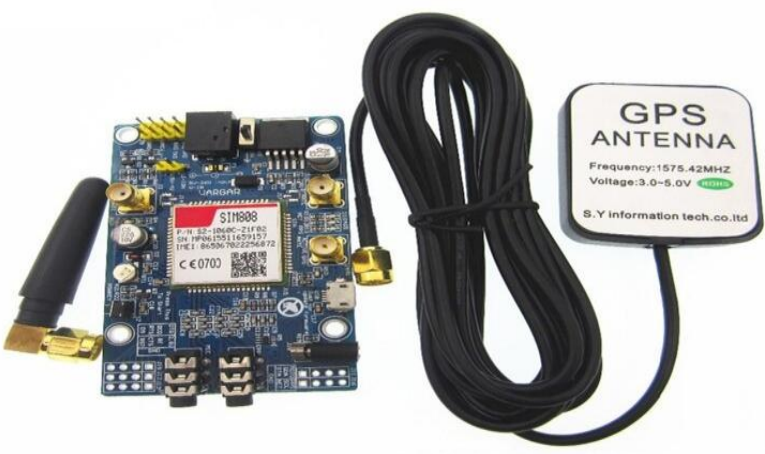

Fig 5: GPS Module

E. Buzzer

A buzzer is a audio signaling module, which can be mechanical, electro-mechanical, or sometimes may be piezoelectric (piezo means short). Common uses of buzzers or beepers include alarms, etc.

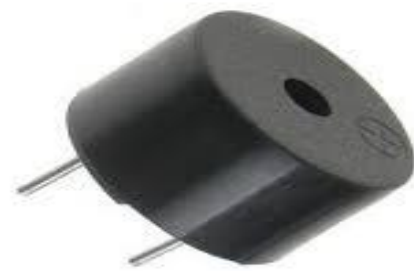

Fig 6: Buzzer

\section{DATA FLOW DIAGRAM}

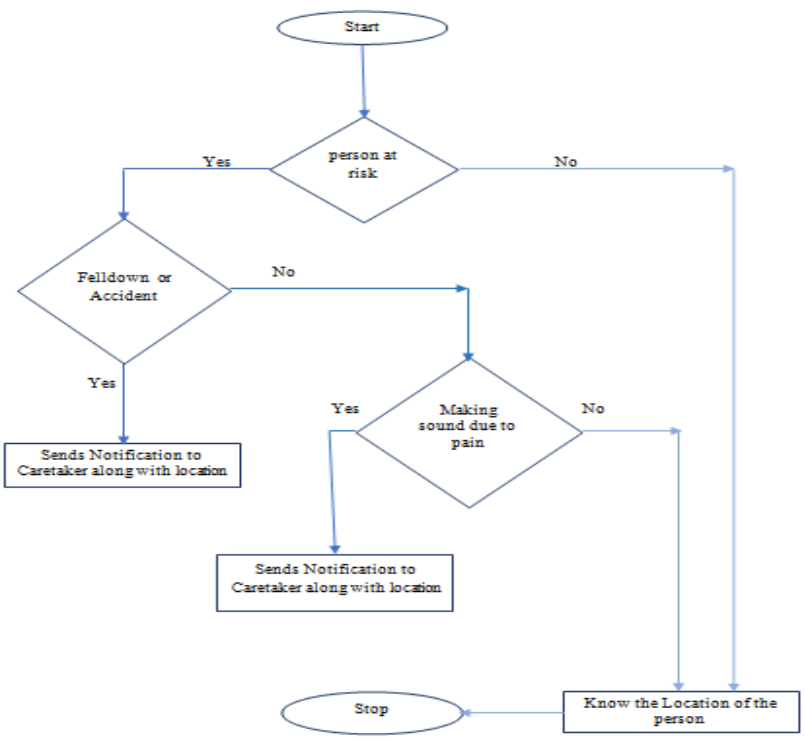

Fig 7: Flow chart depicting dataflow

Published By:

Blue Eyes Intelligence Engineering \& Sciences Publication

(c) Copyright: All rights reserved.

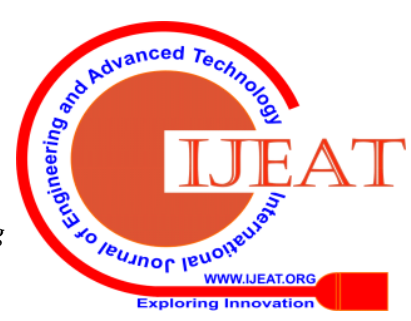


In the above Fig 7 the dataflow of the prototype which is an smart system for elderly wandering person, if person is at risk, that is if he fell down or met with accident or makes sound due to pain our model sends notification to caretaker along with notification.

\section{RESULTS AND DISCUSSION}

The results that the prototype display are as follows:

1)Detects when person falls and send the location of person to caretaker.

2)Detects when person makes noise due to pain.

3)Easily track the person location.

4)There is buzzer to alert the people around the fallen person.

\section{CONCLUSION}

Around two-thirds of all dementia sufferers live in the home alone according to report. It is rising every year and it is projected that between 2010 and 2033 the percentage of people with schizophrenia among the ages of 70 and 85 who live alone will increase to 40 percent. Helping people live independently in a safe and healthy manner is a great task for our community and is also our goal as researchers.

The new system can help elderly people and reduce the risk of mobile phone surveillance going out for shopping or daily walks.

\section{REFERENCES}

1. The M.E. Pollack, "Intelligent technology for an aging population: using AI to support mentally disabled elderly people," AI Magazine, vol. 26 Summer 2005, no. 2, pp. 9-24.

2. K. Laudau, G.K. Auslander, N. Shoval, S. Werner and J. Heinik, "The views of families and caregivers on using new technology to track people with dementia," Qualitative Health Research, vol. 20 March 2010, No. 3, pp. 409-419.

3. E.V. Hebert, J. Weuve, D.A. and P.A. Scherr. Evan, "US projected Alzheimer's disease (2010-2050), using the 2010 census," Neurology, vol. 80, number 9, February 2013, pp. 1778-83. 4 ] H.W. Naumann, J. Hurtienne, P.M. Langdon, S. Gollner and P.J. Clarkson Clarkson, "Technology supporting the everyday life of people with dementia," International Conference on Inclusive Design and Communications, 2011.

4. H. Hiyama, Y. Shimizu, A. Iwata, D. Anzai and J. Wang, "Wearable BLE Antenne Quality Improvement Review," IEICE Technical Report, Vol. 117, 2017, No. 357, pp. 25-30.

5. K. (In Japanese) Akutsu, "Hokou no kagaku," Tokio: Shuppan Humaido, 1975.

6. G. Cipriani, C. Lucetti, A. Nuti, and S. Danti, "Wandering and dementia," Psychogeriatrics, vol. 14, no. 2, pp. 135-142, 2014.

7. A. Mart'inez-Balleaste, P. A. Perrez-Martinez, and A. Solanas, "The pursuit ' of citizens' privacy: A privacy-aware smart city is possible," IEEE Communications Magazine, vol. 51, no. 6, pp. 136-141, 2013.

8. Y.-J. Chango, "Anomaly detection for travelling individuals with cognitive impairments," ACM SIGACCESS Accessibility and Computing, no. 97, pp. 25-32, 2010

\section{AUTHORS PROFILE}

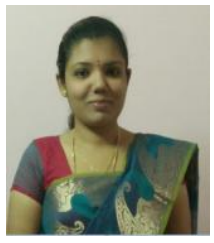

Vidya $\mathbf{J}$ is an Assistant Professor, Department of Computer Science and Engineering at GITAM School of Technology, Bengaluru campus, Karnataka, India. She has done her B.E and M.Tech from R.L. Jalappa Institute of Technology and Channabasaveshwara Institute of Technology, respectively. She is having 3.9 years of teaching experience. She has published 5 international journal papers. Her area of interest include Internet of Things and Machine Learning.

Email-ID-vjyothip@gitam.edu

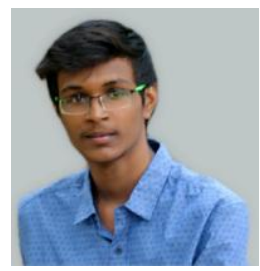

Raja Vineeth pursuing final year B.Tech at GITAM(deemed to be university), Bengaluru. His areas of interest are Data Analysis, Robotic process Automation. He has certifications on Big Data Analytics and Robotic Process Automation. He has done projects on Report Card Generation. Also certified by DELL EMC for Data Analysis certification. He certified as Essential RPA professional by Automation Anywhere. He completed his schooling at Bhashyam English Medium School and higher education at Narayana Junior College.

\section{Email-ID- rajavineeth25041999@gmail.com}

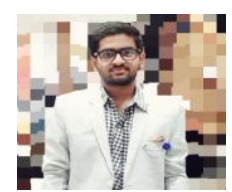

P Sri Harsha pursuing final year B.Tech at GITAM(deemed to be university), Bengaluru. His areas of interest are Data analytics and Artificial robotic processing. He has Certifications on Big data analytics. He has done projects on Heart rate monitoring and Smart system for elderly wandering person. Also certified by DELL EMC for data analysis certification. He completed his schooling at Narayana Techno School and higher education at Aditya Junior College.

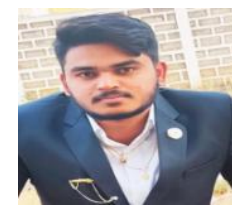

J Venkata Sai pursuing final year B.Tech at GITAM(deemed to be University), Bengaluru. His areas of interest are Data Analysis, Big Data. He has certifications on Big Data Analytics and java. He has done projects on Report Card Generation. Also certified by IBM for c and c++ certification. He completed his schooling at green valley rural School and higher education at Chaitanya Junior College.

Email-ID-venkatasai0078@gmail.com

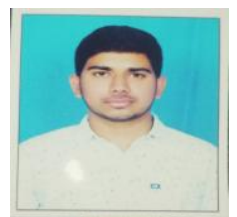

Shaik Noor Mohammed pursuing final year B Tech at GITAM (deemed to be university), BENGALURU. His area of interests are Java full stack developer and python programming. He has done certification on Java programming certified by WEBTEK LABS PVT LTD. He completed his schooling at sri Santhiniketan English Medium School and higher education at Space junior college.

Email-ID- Shaiknoormohammed111@gmail.com

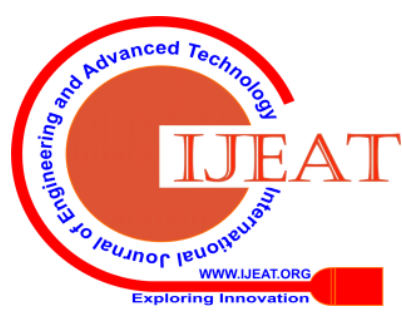

